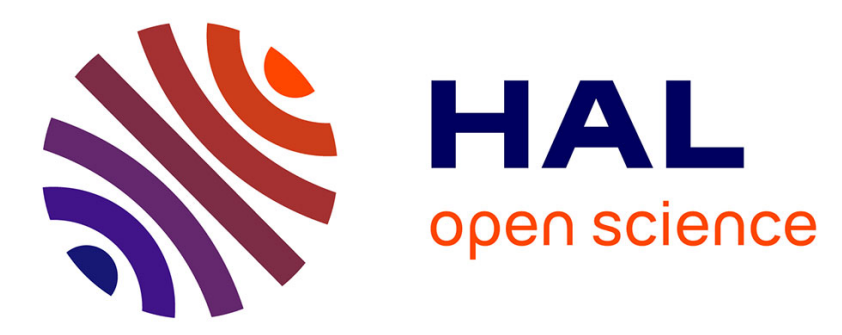

\title{
Terahertz vortex beam generation by infrared vector beam rectification
}

\author{
Ali Al Dhaybi, Jerome Degert, Etienne Brasselet, Emmanuel Abraham, Eric \\ Freysz
}

\section{- To cite this version:}

Ali Al Dhaybi, Jerome Degert, Etienne Brasselet, Emmanuel Abraham, Eric Freysz. Terahertz vortex beam generation by infrared vector beam rectification. Journal of the Optical Society of America B, 2019, 36 (1), pp.12-18. 10.1364/JOSAB.36.000012 . hal-02152636

\section{HAL Id: hal-02152636 \\ https://hal.science/hal-02152636}

Submitted on 11 Jun 2019

HAL is a multi-disciplinary open access archive for the deposit and dissemination of scientific research documents, whether they are published or not. The documents may come from teaching and research institutions in France or abroad, or from public or private research centers.
L'archive ouverte pluridisciplinaire HAL, est destinée au dépôt et à la diffusion de documents scientifiques de niveau recherche, publiés ou non, émanant des établissements d'enseignement et de recherche français ou étrangers, des laboratoires publics ou privés.

\section{(1)(1) $\$(0)$}

Distributed under a Creative Commons Attribution - NonCommercial - ShareAlikel 4.0 


\title{
Terahertz vortex beam generation by infrared vector beam rectification
}

\author{
Ali Al Dhaybi ${ }^{1}$, Jérôme Degert ${ }^{1}$, Etienne Brasselet ${ }^{1}$, Emmanuel Abraham ${ }^{1}{ }^{1,}$, And Eric \\ FREYSZ $^{1}$ \\ ${ }^{1}$ Univ. Bordeaux, CNRS LOMA, UMR 5798, F-33400 Talence, France \\ *Corresponding author: emmanuel.abraham@u-bordeaux.fr
}

Compiled November 8, 2018

\begin{abstract}
We report on the conversion of an infrared vector beam into terahertz vortex beams using a $\langle 110\rangle$-cut ZnTe cubic crystal. First, we provide a theoretical analysis demonstrating how an infrared vector beam with the azimuthal order $\ell$ can be transformed into a terahertz beam endowed with an orbital angular moment content that consists of optical vortices with topological charge $\pm 2 \ell$. Experimentally, quasi-monochromatic terahertz vortex beams with topological charges +2 and -2 are produced and characterized both in amplitude and phase using real-time two-dimensional imaging of the terahertz electric field. These results enrich the terahertz vortex beam toolbox via the transfer of topological information from infrared to terahertz domains. (๑) 2018 Optical Society of America
\end{abstract}

OCIS codes: $\quad$ (080.4865) Optical vortices; (190.0190) Nonlinear optics; (040.2235) Far infrared or terahertz; (110.6795) Terahertz imaging; (260.5430) Polarization; (060.5060) Phase modulation; (070.6110) Spatial filtering.

http://dx.doi.org/10.1364/ao.XX.XXXXXX

\section{INTRODUCTION}

The unique properties of terahertz $(\mathrm{THz})$ radiation, such as good penetration through non-conductive materials and low photon energy, encouraged the development of $\mathrm{THz}$ photonics [1-3]. Besides the development of efficient $\mathrm{THz}$ sources and detectors [4], the progress of $\mathrm{THz}$ optics is also mandatory to enable the specific control and manipulation of $\mathrm{THz}$ radiation, through its amplitude, phase and polarization. A particular situation corresponds to topological beam shaping that refers to tailormade structured fields endowed with phase and/or polarization singularities. Such beams, possibly carrying orbital angular momentum, are now common in the visible and infrared (IR) domains and have already found a huge number of applications (sensing, microscopy and astronomical imaging, trapping and manipulation of matter, information and communication technologies). However, to date, much less works have been reported in the $\mathrm{THz}$ domain.

A basic ingredient of structured light corresponds to phase singularities (or 'vortices') that refer to location in space where the phase is undefined. Many techniques have been implemented to engineer beams carrying on-axis phase singularities - usually called vortex beams - in the visible and IR domains. Quite naturally, a straightforward strategy consisted in mapping these techniques to the $\mathrm{THz}$ domain. One can mention the use of refractive three-dimensional spiral phase plates, with an early demonstration in the sub-THz domain 20 years ago
[5] and more recent realizations covering the $\mathrm{THz}$ domain at $0.3 \mathrm{THz}[6], 0.6 \mathrm{THz}[7,8]$ and in the $2-4 \mathrm{THz}$ range [9]. $\mathrm{THz}$ vortex beam generators have also been realized at $0.75 \mathrm{THz}$ [10], $0.31 \mathrm{THz}$ [11], in the $1-1.6 \mathrm{THz}$ [12] and $0.3-3 \mathrm{THz}$ ranges using metasurface with circular slits [13]. THz vortex beams were generated at $0.15 \mathrm{THz}, 0.3 \mathrm{THz}$ [14-16] and $0.5 \mathrm{THz}$ [17] using three-dimensional printed materials, at $1 \mathrm{THz}$ using a photopatterned liquid crystal [18] or space-variant birefringent slabs [19] and also at 2.1 THz using computer generated holograms [20].

All these works are based on the a direct structuration of the $\mathrm{THz}$ radiation. However, another approach consists in the primary conversion of an optically visible Gaussian beam into a vortex beam and its subsequent conversion into a $\mathrm{THz}$ vortex beam. Pioneering work by Imai et al. consisted in converting a radially polarized IR femtosecond laser beam into a radially polarized $\mathrm{THz}$ beam using an IR segmented waveplate and a $\langle 111\rangle$-cut ZnTe crystal for THz generation by optical rectification [21, 22]. Then, an achromatic THz quarter-wave plate and a polarizer were used to convert this $\mathrm{THz}$ vector beam into a broadband $\mathrm{THz}$ vortex beam with topological charge \pm 1 . However, the direct conversion of the IR vector beam into the $\mathrm{THz}$ vector beam in the ZnTe crystal is only possible if the beams collinearly propagate along the $\langle 111\rangle$ axis of the ZnTe crystal possessing threefold rotational symmetry [23]. Indeed, when the ZnTe crystal is illuminated by linearly polarized IR femtosecond pulses, the emitted $\mathrm{THz}$ pulses are linearly polarized and their 
amplitude remains unchanged as the azimuthal direction $\varphi$ of the incident linearly polarized IR pulses rotates [24]. However, this crystal is not commonly used among the THz community since the $\mathrm{THz}$ generation efficiency is optimized by a factor $2 / \sqrt{3}$ by using a more common $\langle 110\rangle$ ZnTe crystal [23]. We also note that in [22] the theoretical analysis of the conversion of the radially polarized IR beam into the radially polarized $\mathrm{THz}$ beam is not developed.

Here, we present the general mathematical description of $\mathrm{THz}$ vortex beam generation by IR vector beam rectification in a $\langle 110\rangle \mathrm{ZnTe}$ crystal. Then, we perform an experimental demonstration by using radially polarized IR beams, which leads to the generation of generating $\mathrm{THz}$ vortex beams with topological charges \pm 2 .

\section{THEORY}

\section{A. THz electric field generation in a $\langle 110\rangle$ ZnTe crystal}

THz pulse generation by optical rectification of an IR laser pulse in a $\langle 110\rangle$-cut ZnTe crystal is modeled as follows. Both pulses are assumed to propagate along the $\mathbf{x}$-axis in the cartesian frame $(\hat{\mathbf{x}}, \hat{\mathbf{y}}, \hat{\mathbf{z}})$ of the laboratory, which coincides with the $\langle 110\rangle$-axis of the crystal (see Fig. 1). As a consequence, in the frame of the laboratory, the incident IR electric field is written $\mathbf{E}=E_{y} \hat{\mathbf{y}}+$ $E_{z} \hat{\mathbf{z}}$. In the cartesian frame $(\hat{\mathbf{X}}, \hat{\mathbf{Y}}, \hat{\mathbf{Z}})$ of the crystal, the latter expression becomes:

$$
\left(\begin{array}{c}
E_{X} \\
E_{Y} \\
E_{Z}
\end{array}\right)=\mathcal{R} \cdot\left(\begin{array}{c}
0 \\
E_{y} \\
E_{z}
\end{array}\right)
$$

where $\mathcal{R}$ is the matrix associated to the change of basis, defined as:

$$
\mathcal{R}=\left(\begin{array}{ccc}
\frac{1}{\sqrt{2}} & -\frac{\sin \theta}{\sqrt{2}} & \frac{\cos \theta}{\sqrt{2}} \\
\frac{1}{\sqrt{2}} & \frac{\sin \theta}{\sqrt{2}} & -\frac{\cos \theta}{\sqrt{2}} \\
0 & \cos \theta & \sin \theta
\end{array}\right)
$$

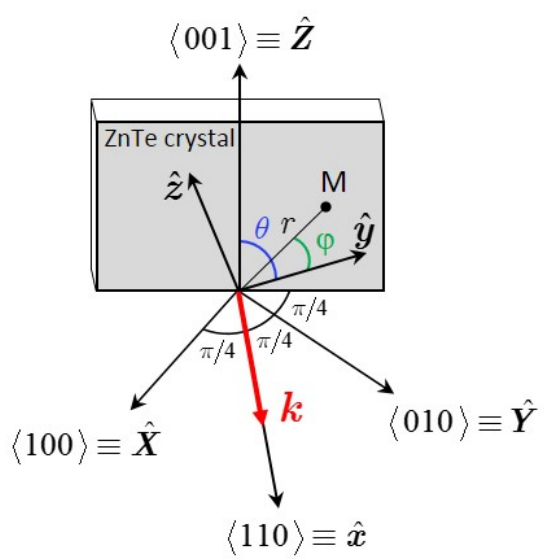

Fig. 1. Geometry of THz generation in the $\langle 110\rangle$ ZnTe crystal. $(\hat{\mathbf{X}}, \hat{\mathbf{Y}}, \hat{\mathbf{Z}})$ : cartesian frame of the crystal; $(\hat{\mathbf{x}}, \hat{\mathbf{y}}, \hat{\mathbf{z}}):$ cartesian frame of the laboratory; $\varphi$ : azimuthal angle; $\theta$ : angle between the horizontal $\mathbf{y}$-axis of the laboratory and the $\mathbf{Z}$-axis of the crystal frame; k: wave vector of the IR pump beam. and $\theta$ is the angle between the horizontal $\mathbf{y}$-axis of the laboratory and the Z Z-axis of the crystal frame, as indicated in Fig. 1. Since $\chi_{X Y Z}^{(2)}$ is the only non-zero component of the second-order nonlinear susceptibility tensor, $\chi^{(2)}$, of the cubic ZnTe crystal [23], the expression of the second-order nonlinear polarization vector, $\mathbf{P}^{(2)}$, in the crystal frame is:

$$
\left(\begin{array}{c}
P_{X}^{(2)} \\
P_{Y}^{(2)} \\
P_{Z}^{(2)}
\end{array}\right)=\varepsilon_{0} \chi^{(2)}: \mathbf{E}^{*}=\varepsilon_{0} \chi_{X Y Z}^{(2)}\left(\begin{array}{c}
E_{Y} E_{Z}^{*} \\
E_{Z} E_{X}^{*} \\
E_{X} E_{Y}^{*}
\end{array}\right)
$$

where * denotes the complex conjugate. This equation involves the multiplication of the input electric field by its complex conjugate. It clearly indicates that the phase factor $\exp (i \ell \varphi)$ of any incoming vortex beam will be canceled out. Consequently, a scalar optical vortex beam cannot be converted into a $\mathrm{THz}$ one through such a nonlinear process.

The situation differs if we consider an incident IR vector beam with azimuthal order $\ell$. In the frame of the laboratory, the corresponding electric field is (omitting the propagation factor and other unimportant phase terms associated with finite beam size):

$$
\mathbf{E}=E_{0}(r)[\cos (\ell \varphi) \hat{\mathbf{y}}+\sin (\ell \varphi) \hat{\mathbf{z}}],
$$

where $r=\sqrt{y^{2}+z^{2}}$ and $\varphi$ are respectively the radius and azimuth of the polar coordinate system in the plane of the crystal, as indicated in Fig. 1, and $E_{0}(r)$ is the Gaussian amplitude of the laser beam. Since the $\mathrm{THz}$ electric field, $\mathbf{E}^{\mathrm{THz}}$, is proportional to $\partial^{2} \mathbf{P}^{(2)} / \partial t^{2}$, combining Eqs. (1)-(4) allows to express, first in the crystal frame and then in the laboratory frame, its angular dependence with respect to $\theta$ and $\varphi$ at the output of the crystal. All calculations done, one finds:

$$
\left(\begin{array}{l}
E_{x}^{\mathrm{THz}} \\
E_{y}^{\mathrm{THz}} \\
E_{z}^{\mathrm{THz}}
\end{array}\right)=E_{0}^{\prime}(r)\left(\begin{array}{c}
0 \\
3 \cos (3 \theta-2 \ell \varphi)-\cos (\theta-2 \ell \varphi)-2 \cos \theta \\
3 \sin (3 \theta-2 \ell \varphi)+\sin (\theta-2 \ell \varphi)-2 \sin \theta
\end{array}\right),
$$

where $E_{0}^{\prime}(r)$ is the Gaussian amplitude of the $\mathrm{THz}$ beam with a $1 / e$ beam waist $w_{\mathrm{THz}}$.

Since the incoming IR laser beam is azimuthally polarized, there is no preferential orientation for the IR electric field in the crystal. Therefore, we can select $\theta=0$ without lack of generality. In that case, the components of the $\mathrm{THz}$ electric field in the frame of the laboratory become:

$$
\begin{aligned}
\left(\begin{array}{c}
E_{x}^{\mathrm{THz}} \\
E_{y}^{\mathrm{THz}} \\
E_{z}^{\mathrm{THz}}
\end{array}\right) & =-\frac{1}{2} E_{0}^{\prime}(r)\left(\begin{array}{c}
0 \\
\sin ^{2}(\ell \varphi) \\
\sin (2 \ell \varphi)
\end{array}\right) \\
& =-\frac{1}{4} E_{0}^{\prime}(r)\left(\begin{array}{c}
0 \\
1-\frac{e^{2 i \ell \varphi}+e^{-2 i \ell \varphi}}{2} \\
\frac{e^{2 i \ell \varphi}-e^{-2 i \ell \varphi}}{i}
\end{array}\right),
\end{aligned}
$$

which reveals that the output of the $\mathrm{THz}$ electric field is a combination of three terms with topological charges $0,2 \ell$ and $-2 \ell$, respectively. 

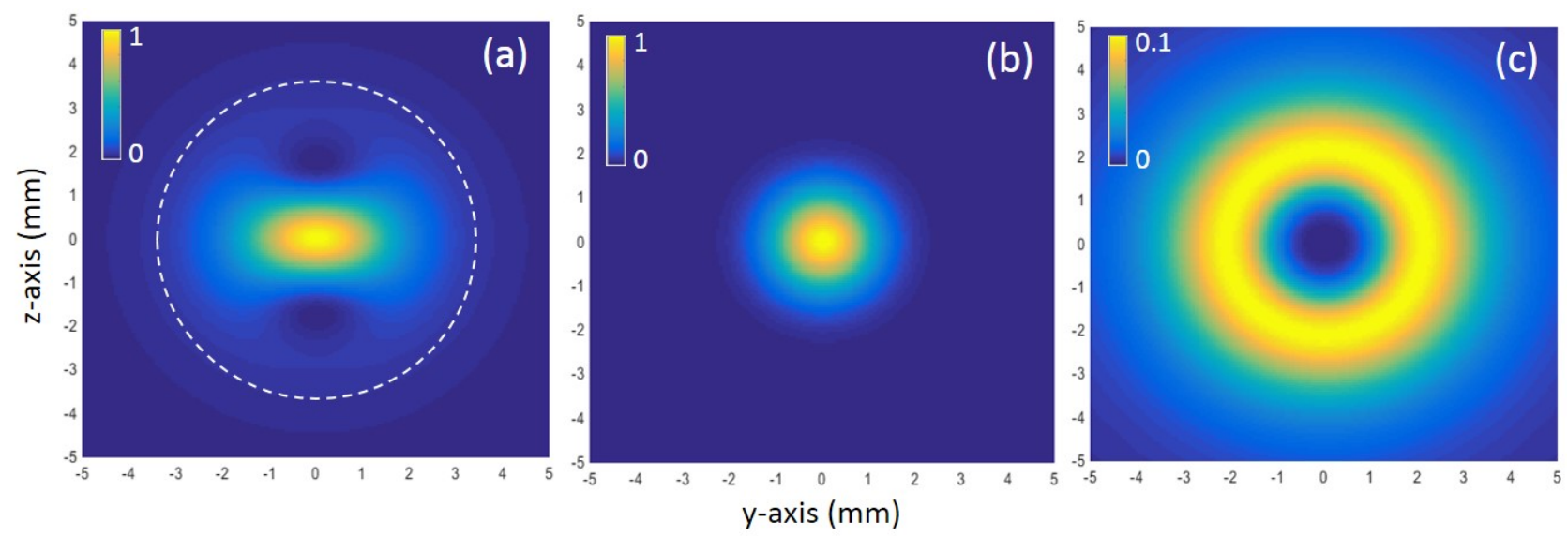

Fig. 2. Numerical simulation of the THz intensity distribution in the Fourier plane. (a) Superposition of a Gaussian beam (topological charge 0) and a vortex beam with topological charge \pm 2 , (Eq. (6)). The dotted circle indicates the size of the mask (see section 3); (b) Intensity distribution of the Gaussian field contribution; (c) Intensity distribution of the vortex field contribution. The physical parameters of the simulation are indicated in the text.

\section{B. Topological charge partial filtering by polarization projec- tion}

Here, we show how one can remove either the topological charge $+2 \ell$ or $2 \ell$ via appropriate polarization projection, by using a quarter-wave plate and a polarizer. After passing through a quarter-wave plate with its fast axis parallel to the $\mathbf{y}$-axis followed by a polarizer whose transmission axis is oriented at an angle $\alpha$ from the $\mathbf{y}$-axis, the THz electric field is polarized in the $\alpha$ direction and expresses as:

$$
E_{\alpha}^{\mathrm{THz}}=E_{y}^{\mathrm{THz}} \cos \alpha+i E_{z}^{\mathrm{THz}} \sin \alpha .
$$

Using Eq. (5), this equation becomes:

$$
\begin{aligned}
E_{\alpha}^{\mathrm{THz}}=-\frac{1}{4} E_{0}^{\prime}(r) & {\left[\cos \alpha+\left(\sin \alpha-\frac{\cos \alpha}{2}\right) e^{2 i \ell \varphi}\right.} \\
& \left.-\left(\sin \alpha+\frac{\cos \alpha}{2}\right) e^{-2 i \ell \varphi}\right]
\end{aligned}
$$

By choosing $\alpha= \pm \alpha^{*}$ such as $\tan \left( \pm \alpha^{*}\right)= \pm 1 / 2$ (i.e. $\alpha^{*} \simeq$ $0.46 \mathrm{rad})$, the vortex component $\pm 2 \ell$ is canceled. Namely,

$$
E_{ \pm \alpha^{*}}^{\mathrm{THz}}=\frac{1}{2 \sqrt{5}} E_{0}^{\prime}(r)\left(1-e^{\mp 2 i \ell \varphi}\right) .
$$

In both cases, the projection of the $\mathrm{THz}$ electric field along the polarizer axis is the superposition of a Gaussian field (topological charge $\ell=0$ ) and a vortex field with topological charge $\pm 2 \ell$.

\section{THz vortex beam extraction by Fourier spatial filtering}

According to Eq. (6), there is an option to extract the $\mathrm{THz}$ vortex beam with a topological charge $+2 \ell$ or $-2 \ell$ through spatial filtering. To simulate this process, we consider a $\mathrm{THz}$ beam with $w_{\mathrm{THz}}=3 \mathrm{~mm}$ and a high-pass spatial filter consisting in a circular metallic dark mask, with radius $R$, placed at the center of the Fourier plane which lies in the second focal plane of a $f=50 \mathrm{~mm}$ lens in a $2 f$ configuration.

Before designing this mask, we need to analyze the distribution of the $\mathrm{THz}$ intensity in the Fourier plane, represented by the square modulus of the two-dimensional Fourier transform of the total THz electric field (see Fig. 2(a)) at $1 \mathrm{THz}$ and for $\ell=1$ ). As expected, the spatial intensity distribution is non-symmetric since the Gaussian and vortex fields mutually interfere in the Fourier plane. It is instructive to plot separately the intensity distributions of the two latter contributions (see Figs. 2(b) and 2(c)). The doughnut-shaped intensity pattern of the vortex has a diameter of $4.2 \mathrm{~mm}$ at its maximum of intensity and a maximum intensity about 10 times smaller than that of the Gaussian beam.

The next step is the selection of the opaque mask. To this end, we used the basic optical arrangement composed of two lenses in a classical $4 f$ architecture. This setup is shown in Fig. 5 and will be detailed later. The evolution of the $\mathrm{THz}$ intensity and phase as a function of $R$ are presented in Fig. 3(a) and Fig. 3(b), respectively, with the parameters $w_{\mathrm{THz}}=3 \mathrm{~mm}$, $\ell=1$ and $\alpha=-\alpha^{*}$. Without the mask $(R=0)$, the intensity distribution is simply the square modulus of the $\mathrm{THz}$ electric field, characterized by Eq. (6). The intensity is inhomogeneous with an azimuthal dependence governed by the factor $\sin ^{2} \varphi$. The phase distribution increases linearly as a function of the azimuthal angle $\varphi$ with a slope +1 . Then, from $R=1 \mathrm{~mm}$ to $R=$ $3.5 \mathrm{~mm}$, the intensity distribution is progressively transformed into an homogeneous Airy disk with diffraction rings slightly visible for $R=3.5 \mathrm{~mm}$. The diffraction effects induced by the mask are also clearly visible with the appearance of $\pi$-shifted circular rings in Fig. 3(b). Morevover, for $R=3.5 \mathrm{~mm}$, inside the central Airy disk, we notice that the phase distribution increases linearly with a slope +2 as a function of the azimutal angle $\varphi$, which emphasizes the almost complete selection of a vortex beam with topological charge +2 .

More quantitatively, we estimate the efficiency and purity of the latter vortex beam extraction as a function of $R$. To this end, we define $P_{\text {in }}$ as the total incident optical power:

$$
P_{\text {in }}=\int_{0}^{\infty}\left|E_{ \pm \alpha^{*}}^{\mathrm{THz}}\right|^{2} 2 \pi r \mathrm{~d} r
$$

with $E_{ \pm \alpha^{*}}^{\mathrm{THz}}$ the electric field defined in Eq. (6). Then, we define $P_{\text {out }}(R)$ as the output power after Fourier filtering:

$$
P_{\text {out }}(R)=\int_{0}^{\infty}\left|\mathcal{F}^{-1}\left[\mathcal{F}\left(E_{ \pm \alpha^{*}}^{\mathrm{THz}}\right) \cdot T_{R}\right]\right|^{2} 2 \pi r \mathrm{~d} r
$$

where $\mathcal{F}$ is the direct two-dimensional Fourier transform, $\mathcal{F}^{-1}$ the corresponding inverse transform and $T_{R}$ the transmittance 


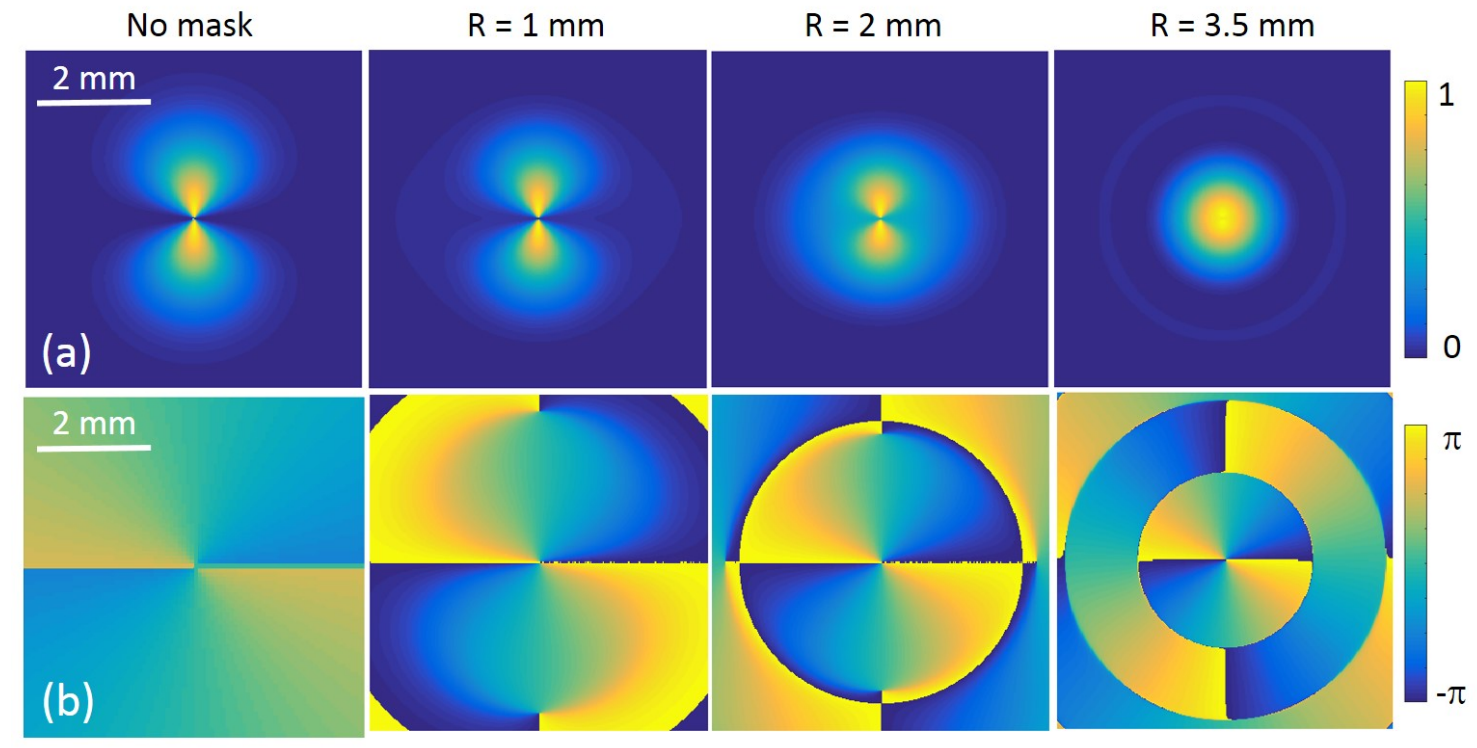

Fig. 3. Numerical simulation of THz intensity (a) and phase (b) distribution after spatial filtering with a circular mask with radius $R$. For a better representation, THz intensity is multiply by the factors 1.33 for $R=1 \mathrm{~mm}, 2.8$ for $R=2 \mathrm{~mm}$ and 4 for $R=3.5 \mathrm{~mm}$, respectively. The physical parameters of the simulation are indicated in the text.

of the mask in the Fourier plane, namely $T_{R}=0$ for $r \leq R$ and $T_{R}=1$ for $r>R$. The ratio $P_{\text {out }} / P_{\text {in }}$ is plotted in Fig. 4 as a function of $R$ (blue curve). This curve emphasizes the drastic decrease of the power efficiency of the spatial filtering process as the mask radius increases. On the other hand, the vortex purity $p(R)$ is defined as the ratio between the optical power transmitted by the spatial filtering for a pure incoming vortex beam with topological charge $\pm 2 \ell$ and $P_{\text {out }}(R)$. Thus:

$$
p(R)=\frac{\int_{0}^{\infty}\left|\mathcal{F}^{-1}\left[\mathcal{F}\left(E_{0}^{\prime}(r) e^{\mp 2 i l \varphi}\right) \cdot T_{R}\right]\right|^{2} 2 \pi r d r}{P_{\text {out }}(R)} .
$$

The evolution of $p(R)$ is indicated by the red curve in Fig. 4. It indicates that $p(R)$ equals $0.5,0.68,0.95$ and 0.999 for $R$ equals 0 , 1,2 and $3.5 \mathrm{~mm}$, respectively. It is then a question of signal-tonoise ratio of the detection to select the optimized value of $R$ for the experiment. In our case, as presented in the next section, we selected $R=3.5 \mathrm{~mm}$.

\section{RESULTS AND DISCUSSION}

\section{A. Experimental setup}

In practice, linearly polarized femtosecond IR laser pulses ( $800 \mathrm{~nm}, 1 \mathrm{~mJ}, 35 \mathrm{fs}, 1 \mathrm{kHz}$ repetition rate, $3 \mathrm{~mm}$ beam waist) were sent onto a Vector Vortex Waveplate (VVW) from Beamco (Fig. (5). The functional layer of this plate is a liquid crystal polymer whose thickness and space variant in-plane orientation of the optical axis allows generating a radially polarized IR laser beam at $800 \mathrm{~nm}$ (see Eq. (4) with $\ell=1$ ). Then, the IR vector beam is sent onto a $\langle 110\rangle$ ZnTe crystal for optical rectification of the femtosecond IR laser pulses and broadband $\mathrm{THz}$ generation $(0.1-3 \mathrm{THz})$. The nonlinear crystal is $1 \mathrm{~mm}$-thick with a clear aperture of $25 \mathrm{~mm}$. After passing through the monochromatic $1 \mathrm{THz}$ quarter-wave plate and the polarizer $\mathrm{P}$, the $\mathrm{THz}$ beam is focused and then recollimated by a standard 1:1 afocal optical arrangement composed of two THz lenses L with $f=50 \mathrm{~mm}$ focal length. The circular metallic mask M, with radius $R$, is

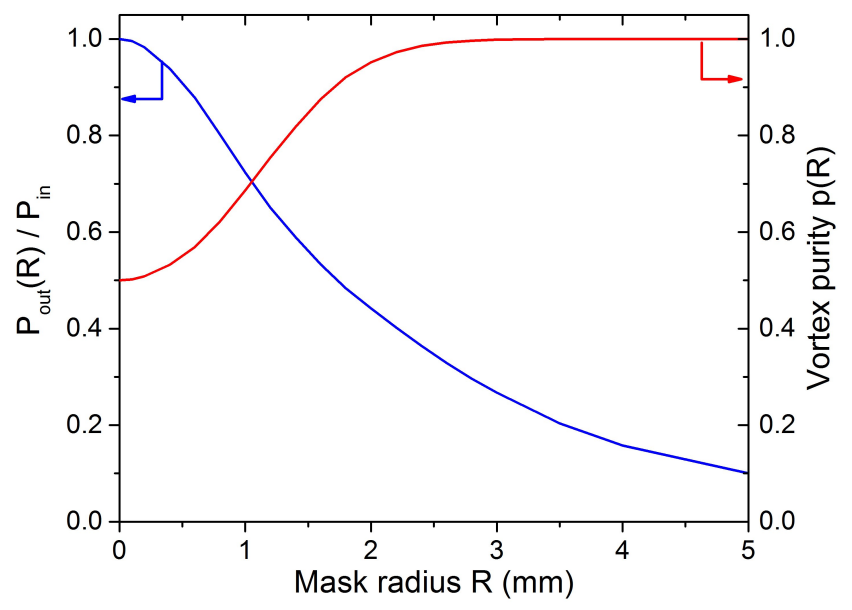

Fig. 4. Power efficiency $P_{\text {out }} / P_{\text {in }}$ (blue curve) and vortex purity $p(R)$ (red curve) as a function of the mask radius $R$.

centered in the second focal plane of the first lens (the 'Fourier plane') for spatial filtering. Finally, the broadband THz electric field is spectrally filtered at $1 \mathrm{THz}$ by the bandpass filter $\mathrm{F}$ before being measured.

The two-dimensional $\mathrm{THz}$ electric sensor has been described in $[19,25,26]$. Briefly, the THz beam is sent into a second $1 \mathrm{~mm}$ thick $\langle 110\rangle$ ZnTe crystal. Owing to a collinear electro-optic (EO) detection with a time-delayed femtosecond laser probe pulse reflected by the front face (left side) of the crystal [27], we are able to convert the $\mathrm{THz}$ electric field distribution onto the spatial profile of the IR laser probe beam which is detected by a CMOS camera. The objective lens located in front of the camera conjugates optically the second $\mathrm{ZnTe}$ crystal ( $\mathrm{THz}$ detector) with the CMOS chip. Consequently, since the EO detection enables the determination of both amplitude and phase of the THz electric field, our experimental setup gives the spatial distribution of the 


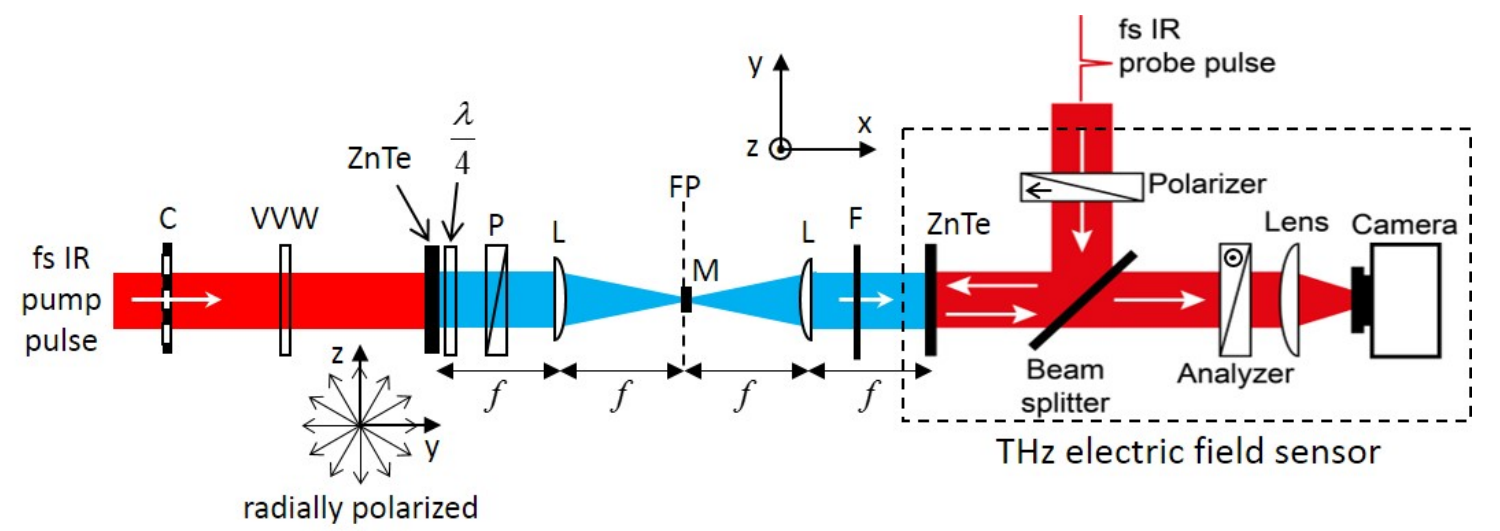

IR field

Fig. 5. Experimental setup. C: chopper; VVW: Vector Vortex Waveplate; $\lambda / 4$ : quarter-wave plate at $1 \mathrm{THz}$; $\mathrm{P}$ THz polarizer; L: plano-convex THz lens with $f=50 \mathrm{~mm}$ focal length; FP: Fourier plane; F: $1 \mathrm{THz}$ bandpass filter; M: mask.

THz amplitude and phase at the output of the first ZnTe crystal (THz emitter). More precisely, for a given time delay $t$ between the $\mathrm{THz}$ and the probe pulses, the system is able to provide a two-dimensional image, at $800 \mathrm{~nm}$, corresponding to the distribution of the THz electric field. This image can be obtained with a $500 \mathrm{~Hz}$ acquisition rate thanks to the synchronization with the laser repetition rate and a dynamic subtraction method [28].

The experimental procedure to measure the THz electric field has been detailed in [29]. Shortly, for every pixels of the camera, i.e. for all $y$ and $z$ values in the ZnTe detection crystal plane, it is possible to plot the $\mathrm{THz}$ temporal waveform. Then, through a Fourier transform of the temporal data, it is possible to calculate the amplitude and the phase of the complex THz electric field. In our experiment, the $\mathrm{THz}$ spectrum ranges from 0 to $3 \mathrm{THz}$ [29]. It is next straightforward to plot the amplitude and phase of the THz electric field at a given frequency and obtain the final wavefront from the phase map, characterized by the identical phase surface of the electromagnetic field.

\section{B. Results}

First, after Fourier transform of the temporal data, we measure the spectral amplitude and phase distributions of a reference beam at $1 \mathrm{THz}$ in the absence of VVW, $\lambda / 4, \mathrm{P}$ and $\mathrm{M}$. In that case, the $\mathrm{THz}$ generation is optimized by selecting the crystal orientation angle $\theta=0.95 \mathrm{rad}$ [23]. The electric field amplitude at $1 \mathrm{THz}$ is $\mathbf{y}$-polarized with a Gaussian-like transverse distribution, as shown in Fig. 6(a). The beam radius is estimated as $w_{\mathrm{THz}}=3 \mathrm{~mm}$, which corresponds to the value used in section 2 . We assume that the spatial inhomogeneity of the $\mathrm{THz}$ amplitude is due to the incoming pump laser beam and the optical rectification process in the first ZnTe crystal. Similarly, Fig. 6(b) shows the $\mathrm{THz}$ wavefront at $1 \mathrm{THz}$. As expected, it is nearly planar in the central part of the beam with negligible geometrical aberrations [29]. The temporal evolution of the THz electric field, at the position indicated by the black cross in Figs. 6(a) and (b), is shown in Fig. 6(c). As expected, the THz waveform is a $T=1 \mathrm{ps}$ pseudo-periodic waveform. The Fourier transform of the latter gives a $\mathrm{THz}$ spectrum with a narrow peak at $1 \mathrm{THz}$, as shown in Fig. 6(d).

Then, we inserted the VVW, the $1 \mathrm{THz}$ quarter-wave plate with its fast axis parallel to the $\mathbf{y}$-axis and the polarizer whose transmission axis is oriented at the angle $\alpha=-\alpha^{*}$. In that case, according to our theoretical analysis, the projection of the $\mathrm{THz}$
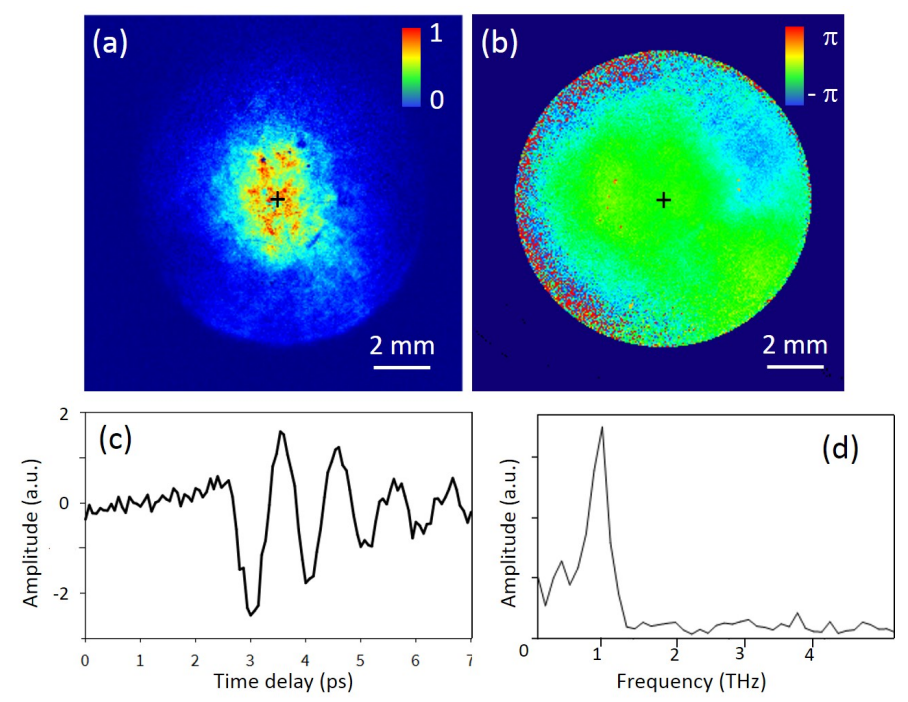

Fig. 6. Reference $\mathrm{THz}$ electric field distribution obtained without VVW, THz quarter-wave plate, polarizer and mask. (a) Amplitude at $1 \mathrm{THz}$; (b) Phase at $1 \mathrm{THz}$; (c) Temporal waveform at the position indicated by the black cross in (a) and (b); (d) Corresponding spectrum.

electric field along the polarizer axis gives the superposition of topological charges 0 and +2 given by Eq. (6). For our experiment, we selected $R=3.5 \mathrm{~mm}$ for spatial filtering of the incoming wave. For this value, as shown in Fig. 4, the remaining beam power represents $20 \%$ of the incident beam power and the vortex purity is almost 1. Fig. 7(a) shows the temporal evolution of the THz electric field distribution for different delay times $t$ between the IR probe pulse and the THz signal. As expected, from $t=0$ to $t=T=1 \mathrm{ps}$, the spatiotemporal spiraling structure of the electric field corresponds to the presence of a phase singularity. After Fourier transform of the temporal data, we can obtain the intensity (Fig. 7(b)) and the phase (Fig. 7(c)) of the $1 \mathrm{THz}$ electric field. The roughly doughnut-shaped intensity pattern is due to optical imaging misalignment and residual diffraction effects of the experimental setup. It is reminiscent of the on-axis phase singularity that is associated to a zero of $\mathrm{THz}$ intensity. The linear azimuthal dependence of the phase 

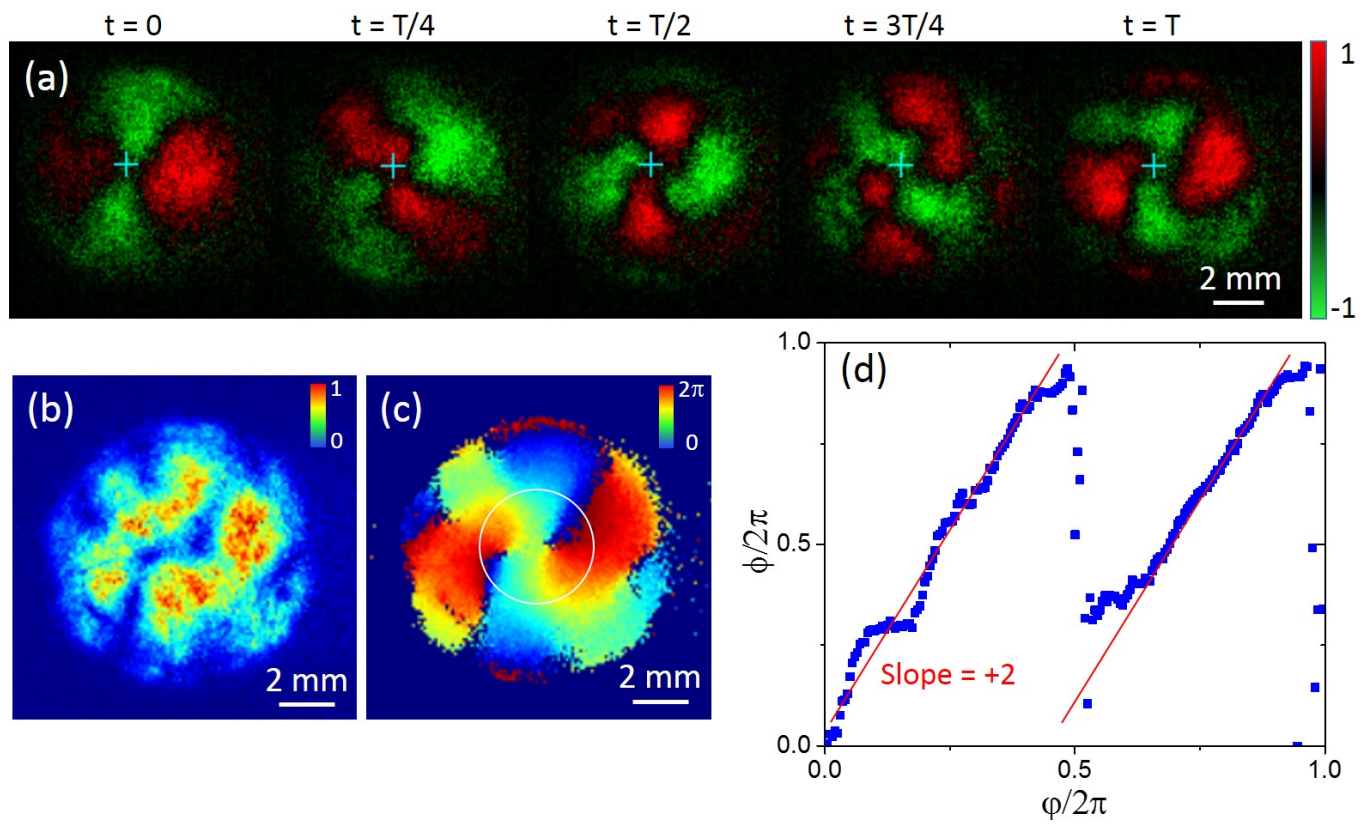

Fig. 7. $\mathrm{THz}$ vortex beam with topological charge +2 . (a) Temporal evolution of the $\mathrm{THz}$ electric field distribution. $\mathrm{T}=1 \mathrm{ps}$; (b) Amplitude of the $1 \mathrm{THz}$ electric field distribution, after Fourier transform of the temporal data; (c) Phase of the $1 \mathrm{THz}$ electric field distribution, after Fourier transform of the temporal data; $(\mathrm{d})$ Evolution of the phase as a function of the azimuthal angle $\varphi$. The experimental points corresponds to the phase values along the white circle in (d).

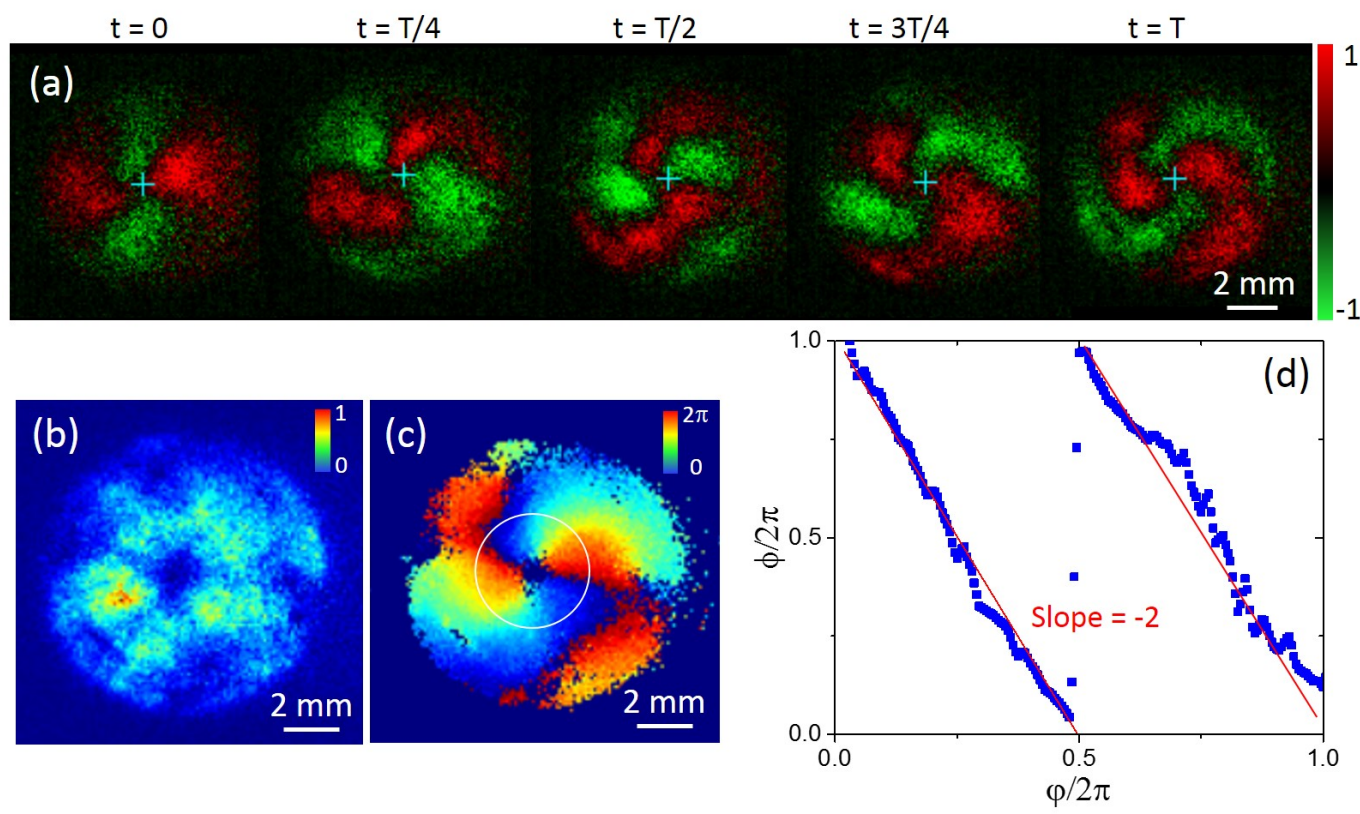

Fig. 8. THz vortex beam with topological charge -2 . (a) Temporal evolution of the THz electric field distribution. $T=1$ ps; (b) Amplitude of the $1 \mathrm{THz}$ electric field distribution, after Fourier transform of the temporal data; (c) Phase of the $1 \mathrm{THz}$ electric field distribution, after Fourier transform of the temporal data; (d) Evolution of the phase as a function of the azimuthal angle $\varphi$. The experimental points corresponds to the phase values along the white circle in (d).

is observed in Fig. 7(c) and quantitatively shown in Fig. 7(d), which represents the azimuthal evolution of the phase $\phi$ along the white circle in Fig. 7(c). As expected, the phase is linear with a slope +2 , demonstrating the generation of a $\mathrm{THz}$ vortex beam with topological charge +2 , .

Similarly, Fig. 8 shows the experimental result for $\alpha=\alpha^{*}$. In that case, the projection of the THz electric field along the polar- izer axis gives the superposition of topological charges 0 and -2 given by Eq. (6). With the same mask as before $(R=3.5 \mathrm{~mm})$, we generated a $\mathrm{THz}$ vortex beam with opposite topological charge -2 , as clearly indicated by the linear phase with a slope -2 in Fig. 8(d). This additional result demonstrates that the sign of the topological charge is actually controlled by changing the polarizer angle from $\alpha=\alpha^{*}$ to $\alpha=-\alpha^{*}$. 


\section{CONCLUSION}

Extending the pioneering work of Imai et al. [22], we demonstrated the generation of a $\mathrm{THz}$ vortex through optical rectification of femtosecond radially polarized IR pulses. With a specific optical arrangement composed of a VVW with azimuthal order $\ell$, a $\langle 110\rangle$-cut ZnTe cubic crystal, a quarter-wave plate, a polarizer and Fourier spatial filtering, we theoretically demonstrated that broadband vortex beams can be generated with topological charges $\pm 2 \ell$. Experimentally, monochromatic vortex beams were generated and characterized at $1 \mathrm{THz}$, with topological charges \pm 2 , depending on the orientation of the polarizer. More generally, our method can also produce broadband $\mathrm{THz}$ vortices by using achromatic polarizing elements such as a silicon Fresnel-rhomb prism. This work enriches the spin-orbit $\mathrm{THz}$ toolbox to prepare structured light endowed with polarization-controlled orbital angular momentum content.

Acknowledgment. The Conseil Régional Nouvelle Aquitaine and FEDER are thanked for funding the equipments of the COLA platform at LOMA.

\section{REFERENCES}

1. W. L. Chan, J. Deibel, and D. M. Mittleman, "Imaging with terahertz radiation," Reports on Progress in Physics 70, 1325-1379 (2007).

2. P. Jepsen, D. Cooke, and M. Koch, "Terahertz spectroscopy and imaging - Modern techniques and applications," Laser \& Photonics Reviews 5, 124-166 (2011).

3. "Terahertz optics," Special issue of Nature Photonics 7, 665-760 (2013).

4. R. A. Lewis, "A review of terahertz sources," Journal of Physics D: Applied Physics 47, 374001 (2014).

5. G. Turnbull, D. Robertson, G. Smith, L. Allen, and M. Padgett, "The generation of free-space laguerre-gaussian modes at millimetre-wave frequencies by use of a spiral phase plate," Opt. Commun. 127, 183-188 (1996).

6. X. Wei, C. Liu, L. Niu, Z. Zhang, K. Wang, Z. Yang, and J. Liu, "Generation of arbitrary order bessel beams via $3 \mathrm{~d}$ printed axicons at the terahertz frequency range," Appl. Opt. 54, 10641-10649 (2015).

7. X. Wang, J. Shi, W. Sun, S. Feng, P. Han, J. Ye, and Y. Zhang, "Longitudinal field characterization of converging terahertz vortices with linear and circular polarizations," Opt. Express 24, 7178-7190 (2016).

8. K. Miyamoto, B. J. Kang, W. T. Kim, Y. Sasaki, H. Niinomi, K. Suizu, F. Rotermund, and T. Omatsu, "Highly intense monocycle terahertz vortex generation by utilizing a Tsurupica spiral phase plate," Scientific Reports 6 (2016).

9. K. Miyamoto, K. Suizu, T. Akiba, and T. Omatsu, "Direct observation of the topological charge of a terahertz vortex beam generated by a Tsurupica spiral phase plate," Applied Physics Letters 104, 261104 (2014).

10. J. He, X. Wang, D. Hu, J. Ye, S. Feng, Q. Kan, and Y. Zhang, "Generation and evolution of the terahertz vortex beam," Optics Express 21, 20230 (2013).

11. J. Salo, J. Meltaus, E. Noponen, M. M. Salomaa, A. Lönnqvist, T. Koskinen, Ville Viikari, J. Säily, J. Häkli, J. Ala-Laurinaho, J. Mallat, and A. V. Räisänen, "Holograms for shaping radio-wave fields," J. Opt. A: Pure Appl. Opt. 4, S161-S167 (2002).

12. Z. Xie, X. Wang, J. Ye, S. Feng, W. Sun, T. Akalin, and Y. Zhang, "Spatial terahertz modulator," Sci. Rep. 3, 3347 (2013).

13. H. Zhou, J. Dong, S. Yan, Y. Zhou, and X. Zhang, "Generation of Terahertz Vortices Using Metasurface With Circular Slits," IEEE Photonics Journal 6, 1-7 (2014).

14. X. Wei, C. Liu, L. Niu, Z. Zhang, K. Wang, Z. Yang, and J. Liu, "Generation of arbitrary order Bessel beams via $3 d$ printed axicons at the terahertz frequency range," Applied Optics 54, 10641 (2015).

15. A. I. Hernandez-Serrano, E. Castro-Camus, and D. Lopez-Mago, "q- plate for the Generation of Terahertz Cylindrical Vector Beams Fabricated by 3d Printing," Journal of Infrared, Millimeter, and Terahertz Waves (2017)

16. C. Liu, J. Liu, L. Niu, X. Wei, K. Wang, and Z. Yang, "Terahertz circular Airy vortex beams," Scientific Reports 7 (2017).

17. Z. Wu, X. Wang, W. Sun, S. Feng, P. Han, J. Ye, Y. Yu, and Y. Zhang, "Vectorial diffraction properties of THz vortex Bessel beams," Optics Express 26, 1506 (2018)

18. S. Ge, P. Chen, Z. Shen, W. Sun, X. Wang, W. Hu, Y. Zhang, and Y. Lu, "Terahertz vortex beam generator based on a photopatterned large birefringence liquid crystal," Optics Express 25, 12349 (2017).

19. A. Minasyan, C. Trovato, J. Degert, E. Freysz, E. Brasselet, and E. Abraham, "Geometric phase shaping of terahertz vortex beams," Optics Letters 42, 41 (2017).

20. B. Knyazev, Y. Choporova, M. Mitkov, V. Pavelyev, and B. Volodkin, "Generation of Terahertz Surface Plasmon Polaritons Using Nondiffractive Bessel Beams with Orbital Angular Momentum," Physical Review Letters 115 (2015).

21. R. Imai, N. Kanda, T. Higuchi, Z. Zheng, K. Konishi, and M. KuwataGonokami, "Terahertz vector beam generation using segmented nonlinear optical crystals with threefold rotational symmetry," Opt. Express 20, 21896-21904 (2012).

22. R. Imai, N. Kanda, T. Higuchi, K. Konishi, and M. Kuwata-Gonokami, "Generation of broadband terahertz vortex beams," Optics Letters 39, 3714 (2014).

23. Q. Chen, M. Tani, Z. Jiang, and X.-C. Zhang, "Electro-optic transceivers for terahertz-wave applications," Journal of the Optical Society of America B 18, 823 (2001).

24. T. Higuchi, N. Kanda, H. Tamaru, and M. Kuwata-Gonokami, "Selection rules for light-induced magnetization of a crystal with threefold symmetry: The case of antiferromagnetic nio," Phys. Rev. Lett. 106, 047401 (2011).

25. E. Abraham, H. Cahyadi, M. Brossard, J. Degert, E. Freysz, and T. Yasui, "Development of a wavefront sensor for terahertz pulses," Optics Express 24, 5203 (2016).

26. M. Brossard, J.-F. Sauvage, M. Perrin, and E. Abraham, "Terahertz adaptive optics with a deformable mirror," Optics Letters 43, 1594 (2018).

27. P. C. Planken, H.-K. Nienhuys, H. J. Bakker, and T. Wenckebach, "Measurement and calculation of the orientation dependence of terahertz pulse detection in ZnTe," JOSA B 18, 313-317 (2001).

28. Z. Jiang, X. G. Xu, and X.-C. Zhang, "Improvement of terahertz imaging with a dynamic subtraction technique," Appl. Opt. 39, 2982-2987 (2000).

29. M. Brossard, H. Cahyadi, M. Perrin, J. Degert, E. Freysz, T. Yasui, and E. Abraham, "Direct wavefront measurement of terahertz pulses using two-dimensional electro-optic imaging," IEEE Transactions on Terahertz Science and Technology 7, 741-746 (2017). 